\title{
Combinatorial Game Theory Foundations Applied to Digraph Kernels
}

\author{
Aviezri S. Fraenkel \\ Department of Applied Mathematics and Computer Science \\ Weizmann Institute of Science \\ Rehovot 76100, Israel \\ fraenkel@wisdom.weizmann.ac.il

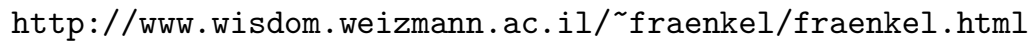

Submitted: August 29, 1996; Accepted: November 21, 1996

To Herb Wilf at the end of the first 5 Bar Mitzvahs: At least 5 more in ever increasing joy and creativity

\begin{abstract}
Known complexity facts: the decision problem of the existence of a kernel in a digraph $G=(V, E)$ is NP-complete; if all of the cycles of $G$ have even length, then $G$ has a kernel; and the question of the number of kernels is \#P-complete even for this restricted class of digraphs. In the opposite direction, we construct game theory tools, of independent interest, concerning strategies in the presence of draw positions, to show how to partition $V$, in $O(|E|)$ time, into 3 subsets $S_{1}, S_{2}, S_{3}$, such that $S_{1}$ lies in all the kernels; $S_{2}$ lies in the complements of all the kernels; and on $S_{3}$ the kernels may be nonunique. Thus, in particular, digraphs with a "large" number of kernels are those in which $S_{3}$ is "large"; possibly $S_{1}=S_{2}=\emptyset$. We also show that $G$ can be decomposed, in $O(|E|)$ time, into two induced subgraphs $G_{1}$, with vertex-set $S_{1} \cup S_{2}$, which has a unique kernel; and $G_{2}$, with vertex-set $S_{3}$, such that any kernel $K$ of $G$ is the union of the kernel of $G_{1}$ and a kernel of $G_{2}$. In particular, $G$ has no kernel if and only if $G_{2}$ has none. Our results hold even for some classes of infinite digraphs.
\end{abstract}




\section{Introduction}

Modern combinatorial game theory has largely been a parasite: it drew tools and results from fields such as logic, computational complexity, graph and matroid theory, combinatorics, algebra and number theory to generate results for itself. More recently, it has also begun to contribute back to some of its benefactors, such as to surreal numbers, a subject created by John Conway [Con1976], and to linear error-correcting codes (which is linear algebra) [CoS1986], [Con1990] [BrP1993], [Fra1996]

In this paper we develop some basic concepts of 2-player game theory, and use them to shed new light on the structure of digraph kernels. Connections between kernels and game-theory have been explored in the past, see e.g. Berge [Ber1992]: the new element here seems to be the use of draw positions for investigating digraph kernels. In fact, the set of draw positions appears to be the "kernel of the kernels", i.e., the part where many of the interesting properties of the kernels are concentrated.

Throughout a digraph is a finite or infinite directed graph, which may contain cycles or loops, unless otherwise specified. A kernel of a digraph $G=(V, E)$ is a subset $K \subseteq V$ which is both independent and dominating. "Independent" means that the subgraph induced by $K$ has no edges (so in particular: no loops); and "dominating" - that every vertex of $V-K$ has a follower (successor) in $K$, i.e., an edge leading into $K$. If $G$ is finite, the decision problem of the existence of a kernel is NP-complete, see Chvátal[Chv1973] and van Leeuwen[[VLe1976] for a general digraph, and Fraenkel [Fra1981] for a planar digraph with indegrees $\leq 2$, outdegrees $\leq 2$ and degrees $\leq 3$. For any tighter constraints the problem is clearly solvable in linear time. It is further known that a finite digraph all of whose cycles have even length has a kernel[Ric1953] and that the question of the number of kernels is \#P-complete even for this restricted class of digraphs [SzC1994]

As an example, consider a digraph with $2 k+1$ vertices and $k$ "blades", as depicted in Fig. 1 for $k=4$. It clearly has $2^{k}$ kernels. The center vertex is in the kernel if and only if all its $k$ followers are not. Note that there is no vertex which is in all the kernels or in the complement of all the kernels for this example.

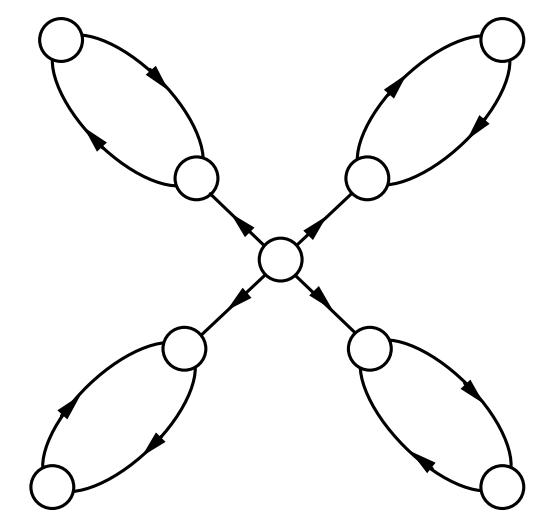

Figure 1 . The case $k=4$ of a digraph with $2 k+1$ vertices and $2^{k}$ kernels. 
Despite all the inefficiency and chaos exuded by these results, we exhibit in this paper a nice structure of digraph kernels; and we show that the inefficiency part can be localized and contained within a well-defined induced subgraph of $G$. Moreover, if $G$ is finite, all of this can be done in $O(|E|)$ steps.

Specifically, we show that for a class of infinite digraphs $G=(V, E)$, there exists a partition of $V$ into 3 subsets $S_{1}, S_{2}, S_{3}$, such that $S_{1}$ lies in all the kernels; $S_{2}$ lies in the complements of all the kernels; and on $S_{3}$ the kernels may be nonunique. For $G$ finite, the partition can be found in $O(|E|)$ steps. In general we cannot be more precise about what happens in $S_{3}$, a region where the NP-completeness reigns, but in special cases one may be able to say something; see e.g., the paragraph after Corollary 5 Note that if a digraph has a "large" number of kernels, then $S_{3}$ must be "large"; possibly $S_{1}=S_{2}=\emptyset$. But $S_{3}$ may be large when there are only few kernels: if $G$ is an $n$-gon, then $S_{3}=V$ and there are $\leq 2$ kernels. Of course $S_{1}=S_{2}=\emptyset$ and $S_{3}=V$ is always a trivial solution, but for many digraphs, especially those with a small number of edges, as found, e.g., in many game-graphs, $S_{3}$ is small. Furthermore, we show that there exists a decomposition of $G$ into two induced subgraphs $G_{1}$, with vertex-set $S_{1} \cup S_{2}$, which has a unique kernel; and $G_{2}$, with vertex-set $S_{3}$, such that any kernel $K$ of $G$ is the union of the kernel of $G_{1}$ and a kernel of $G_{2}$. In particular, $G$ has no kernel (a unique kernel) if and only if $G_{2}$ has no kernel (a unique kernel).

It will also become clear that these results are proved most naturally in a game-theoretic setting; in fact, they can be understood best in terms of the strategy of the following simple coin-pushing game played on $G$. Initially a coin is placed on some vertex of $G$. Two players alternate moves. A move consists of sliding the coin to a follower vertex $v$, which could be $v$ itself, if the move is along a loop ("passing"). The player first unable to move loses, and the other player wins. In the case of an infinite or cyclic digraph, there may be no last move: none of the players can force a win, but each has always a nonlosing move. In this case the outcome is a draw.

The tools from combinatorial game theory, which are of independent interest, concern basic strategies in the presence of possible draw positions, and efficient computational algorithms for implementing them. They appear to be the most natural tools for revealing the structure of digraph kernels. Specifically, we present two equivalent definitions for the losing/winning/drawing-positions in possibly infinite games, and some of their ramifications, including the Fundamental Theorem of Game Theory.

\section{Some Foundational Combinatorial Game Theory}

Combinatorial games, or simply games in the sequel, consist of 2-person games with perfect information (unlike some card games where information is hidden), without chance moves (no dice), and outcome restricted to lose/win, tie/tie and draw/draw for the two players who move alternately. A tie is an end position with no winner and no loser, as may occur in tic-tac-toe for example. A draw is a "dynamic tie", i.e., a non-end position such that neither player can force a win, but each can always find a non-losing move. A position of a game is any state which is reachable in any play of the game, including play with collusion. The play's outcome function is defined on a subset of game positions, called the termination set $\tau$. The player, if any, who first reaches a position in $\tau$ won. The most common convention is normal play, where the player first unable to play loses and the opponent wins, i.e., $\tau$ is the set of end positions; the outcome is reversed in the misère convention. If there is no last move, the outcome is a draw. We restrict our attention to games without ties, because ties behave much like the other outcomes we consider. 
With any game $\Gamma$ we associate a digraph $G=(V, E)$, where $V$ is the set of positions of $\Gamma$ and $(u, v) \in E$ if and only if there is a move from position $u$ to position $v$. It is called the game-graph of $\Gamma$.

Thus any game corresponds to a digraph, namely its game-graph. Conversely, given any digraph $G$, we can define a game whose game-graph is $G$ : initially place a token on any vertex. The 2 players alternate in pushing the token to a follower. Because of this correspondence between digraphs and games, we shall identify games with their corresponding game-graphs, game positions with digraph vertices and game moves with digraph edges, using them interchangeably.

For $u \in V$, the sets

$$
F(u)=\{v \in V:(u, v) \in E\}, \quad F^{-1}(u)=\{w \in V:(w, u) \in E\}
$$

are called the set of followers and the set of predecessors respectively. If $F(u)=\emptyset$, then $u$ is a leaf of $G$.

Informally, given any finite or infinite game $\Gamma$, a P-position is any position $u$ from which "the Previous player can force a win", that is, the opponent of the player moving from $u$ can reach a position in $\tau$ in a finite - though perhaps unbounded - number of moves, independently of the moves of the opponent. An $N$-position is any position $v$ from which "the Next player can force a win", that is, the player who moves from $v$. A D-position is any position from which "a player cannot force a win but has a next nonlosing move". The outcome is then a Draw. The set of all $P, N, D$-positions of a game is denoted by $\mathcal{P}, \mathcal{N}, \mathcal{D}$ respectively.

The game-graph may be infinite, so $|V|$ is a finite or transfinite ordinal. The reader who so prefers can always think of the ordinals in the sequel as being nonnegative integers.

The following is a formal definition of these notions.

Definition 1. Given a game $\Gamma$ without ties which may contain cycles or loops, or may be infinite, with arbitrary termination set $\tau$. Let $G=(V, U)$ be the game-graph of $\Gamma$. Here and in the sequel we denote by $\mathcal{O}$ the set of all ordinals not exceeding $|V|$. By recursion on $n \in \mathcal{O}$ define,

$$
P_{n}=\left\{u \in V: n=\min m, F(u) \subseteq \bigcup_{i<m} N_{i}\right\},
$$

$$
N_{n}=\left\{u \in V: n=\min m, F(u) \cap \bigcup_{i<m} P_{i} \neq \emptyset\right\} .
$$

Finally, we let $\mathcal{P}=\bigcup_{n \in \mathcal{O}} P_{n}, \mathcal{N}=\bigcup_{n \in \mathcal{O}} N_{n}, \mathcal{D}=V \backslash(\mathcal{P} \cup \mathcal{N})$.

Definition 1 doesn't contain any claim about the computational complexity of finding a strategy. We now illustrate Definition 1 on hand of a few examples.

Example 1. Rabin's game [Rab1957] has fixed length 3. It has the form I picks $x_{1}$, II picks $x_{2}$, I picks $x_{3}$. Player I wins if and only if $G\left(x_{1}, x_{2}, x_{3}\right)=0$. The function $G$ is chosen so that player II has a winning strategy, which, however, is not decidable. Other pathological games appear in [Jon1982] and in [JFr1995].

EXAMPLE 2. For the two vertices of Fig. 2(a), the only labels consistent with Definition 1 are $D$; in particular, the labels $P_{0}$ for one and $N_{1}$ for the other are inconsistent with Definition 1 . In Fig. 2(b), the subscripts 0 and 2 of the $P$-positions cannot be interchanged. 


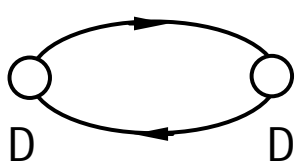

(a)

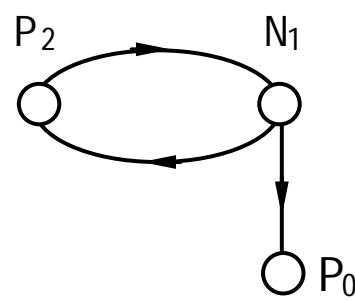

(b)

Figure 2. Games on simple cyclic digraphs.

EXAmple 3. Consider the game $G=(V, E)$ where $V=\mathbb{Z}^{0} \cup\{-1\}$. Every positive integer $m$ has a unique follower $m-1$, and 0 has no follower, so is a leaf; and the followers of -1 are all the positive odd integers. It is easy to see that then $P_{2 i}=\{2 i\}, N_{2 i+1}=\{2 i+1\}$ for all $i \in \mathbb{Z}^{0}$, and $P_{\omega}=\{-1\}$.

EXAmple 4. The game is a digraph $G=(V, E)$, where the vertex set consists of pairs of nonnegative integers, namely, $V=\{(i, j): j \geq 2 i+1, i \in\{0, \ldots, t\}\} \cup\{(0,0)\}$, where $t$ is some fixed positive integer. See Fig. 3 for the case $t=2$. The unique follower of $(i, 2 j)$ is $(i, 2 j-1)$ for $j \geq i+1$. The followers of $(i, 2 j+1)$ for $j \geq i+1$ are $(i, 2 j), i \in\{0, \ldots, t\}$, and $\{(i+1,2 k): k \geq j+1\}$, $i \in\{0, \ldots, t-1\}$. The followers of $(0,0)$ are $\{(0,2 j): j \geq 1\}$. Thus the set of all leaves is $\{(i, 2 i+1): i \in\{0, \ldots, t\}\}$.

Definition 1 implies that

$$
\begin{aligned}
& P_{0}=\{(i, 2 i+1): i \in\{0, \ldots, t\}\}, N_{1}=\{(i, 2 i+2): i \in\{0, \ldots, t\}\}, \\
& P_{2+2 i}=\left\{(t, 2 t+2 i+3): i \in \mathbb{Z}^{0}\right\}, N_{3+2 i}=\left\{(t, 2 t+2 i+4): i \in \mathbb{Z}^{0}\right\}, \\
& P_{\omega+2 i}=\left\{(t-1,2(t-1)+2 i+3): i \in \mathbb{Z}^{0}\right\}, \\
& N_{\omega+2 i+1}=\left\{(t-1,2(t-1)+2 i+4): i \in \mathbb{Z}^{0}\right\}, \\
& P_{\omega 2+2 i}=\left\{(t-2,2(t-2)+2 i+3): i \in \mathbb{Z}^{0}\right\}, \\
& N_{\omega 2+2 i+1}=\left\{(t-2,2(t-2)+2 i+4): i \in \mathbb{Z}^{0}\right\}, \\
& \quad \ldots . \\
& P_{\omega t+2 i}=\left\{(0,2 i+3): i \in \mathbb{Z}^{0}\right\}, N_{\omega t+2 i+1}=\left\{(0,2 i+4): i \in \mathbb{Z}^{0}\right\} .
\end{aligned}
$$

Example 5. The game is as in Example 4, except that there is no bound $t$. Specifically, $V=\left\{(i, j): j \geq 2 i+1, i \in \mathbb{Z}^{0}\right\}$, and the same follower function is defined, except that $i \in \mathbb{Z}^{0}$ instead of the dependence on $t$. The set of leaves is then $P_{0}=\left\{(i, 2 i+1): i \in \mathbb{Z}^{0}\right\}$, and all their predecessors satisfy $N_{1}=\left\{(i, 2(i+1)): i \in \mathbb{Z}^{0}\right\}$. But all the other positions are $D$-positions.

Lemma 1. Let $G=(V, E)$ be a cyclic, possibly infinite, game-graph. Then for every $u \in V$ we have,

$u \in \mathcal{P}$ if and only if $F(u) \subseteq \mathcal{N}$, 


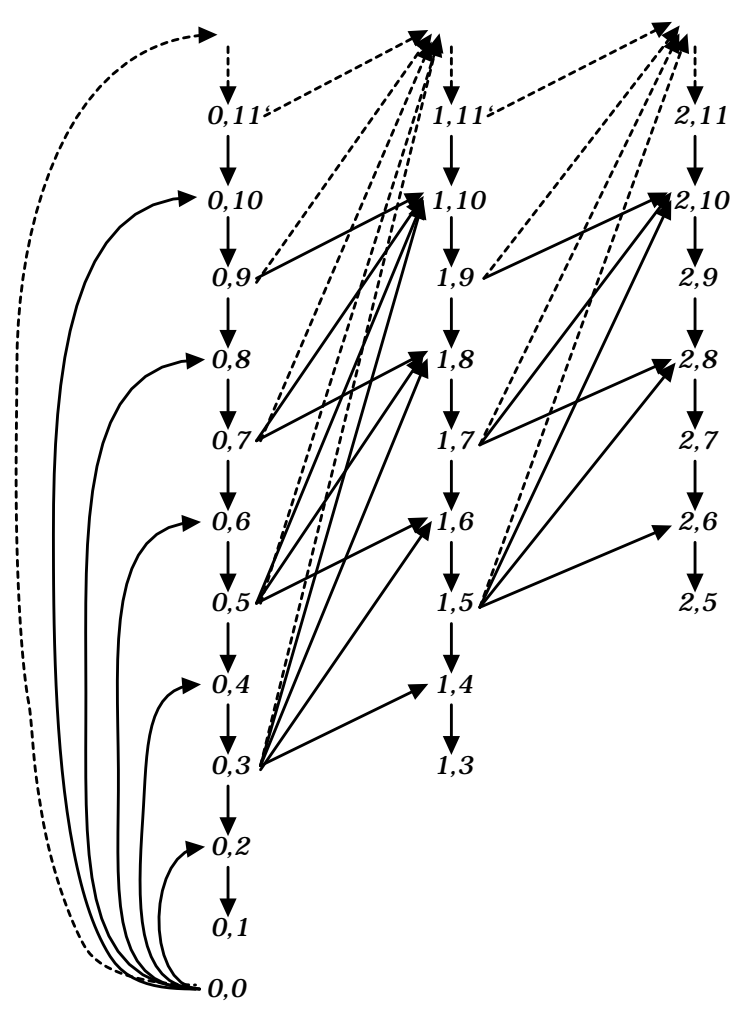

Figure 3. The case $t=2$ of Example 4.

$u \in \mathcal{N}$ if and only if $F(u) \cap \mathcal{P} \neq \emptyset$,

$u \in \mathcal{D}$ if and only if $F(u) \cap \mathcal{P}=\emptyset$ and $F(u) \cap \mathcal{D} \neq \emptyset$.

Proof. Let $u \in \mathcal{P}$. Then $u \in P_{n}$ for some $n \in \mathcal{O}$, so $F(u) \subseteq \bigcup_{i<n} N_{i}$, hence $F(u) \subseteq \mathcal{N}$. The middle part is proved similarly. Now by definition, $u \in \mathcal{D}$ if and only if $u \notin(\mathcal{P} \cup \mathcal{N})$, if and only if $F(u) \cap \mathcal{P}=\emptyset$ (otherwise $u \in \mathcal{N}$ by the second part), and $F(u) \cap \mathcal{D} \neq \emptyset$ (because otherwise $F(u) \subseteq \mathcal{N}$, and so $u \in \mathcal{P}$ by the first part).

Corollary 1. Under the assumptions of Lemma 1, $u \in \mathcal{D}$ if and only if $F(u) \subseteq \mathcal{N} \cup \mathcal{D}$ and $F(u) \cap \mathcal{D} \neq \emptyset$.

Proof. Follows from $F(u) \cap \mathcal{P}=\emptyset$ of Lemma 1 and Definition 1 .

Is it clear that precisely one of the players can always win, or else both can draw, as in the above examples? The answer is given by what we shall call the Fundamental Theorem of Combinatorial Game Theory, analogously to the Fundamental Theorem of Algebra or Arithmetic.

Theorem 1. Let $\Gamma$ be a two-person game with perfect information, no chance moves and no ties ending in lose/win or draw/draw, whose game-graph may be infinite. For any termination set and 
every position of $\Gamma$ there exists a winning move for precisely one of the two players, or else, both players can maintain an infinite sequence of drawing moves, but neither can force a win.

Proof. The last part of Lemma 1 implies that both players can maintain a draw from a $D$ position, but neither can force a win. By Definition 1, each vertex has at least one label in $\mathcal{P} \cup \mathcal{N} \cup \mathcal{D}$. It thus suffices to show that the set $S$ of positions of $\Gamma$ has a unique partition into three subsets $\mathcal{P}$, $\mathcal{N}$ and $\mathcal{D}$.

Suppose $w \in \mathcal{P} \cap \mathcal{N}$. Then let the players play to $w$, possibly using collusion. Starting from $w$, both the Next and the Previous player can win - a contradiction to the only possible outcomes lose/win and draw/draw. We get similar contradictions when assuming $\mathcal{P} \cap \mathcal{D} \neq \emptyset$ or $\mathcal{N} \cap \mathcal{D} \neq \emptyset$.

We point out that Definition 1, Lemma 1 and Theorem 1 are generalizations of previous results by various authors who considered only the outcome (win, lose). Classical theorems of Zermelo [Zer1912] von Neumann[VNe1928] and von Neumann and Morgenstern [VNM1953] state that every finite length 2-person game ends in (lose, win). If, in addition, we restrict attention to games whose length of play is bounded, the proof becomes simpler. See Mark Kac [Kac1974] who attributes the result to Hugo Steinhaus. The result is stated as follows.

Theorem *. Let $G$ be a 2-person game with perfect information, terminating in a bounded number of moves in a win by one of the players. Then there must exist a winning move for either one or the other adversary.

Proof. Denote the moves of players 1 and 2 by $x_{1}, x_{2}, \ldots, x_{n}$ and $y_{1}, y_{2}, \ldots, y_{n}$ respectively. Assuming that player I begins to play, we can express the fact that player 1 has a winning move by,

$$
\left(\exists x_{1}\right)\left(\forall y_{1}\right) \ldots\left(\exists x_{n}\right)\left(\forall y_{n}\right) \quad \text { player I won. }
$$

The negation of this statement is obtained by De Morgan's rule:

$$
\left(\forall x_{1}\right)\left(\exists x_{2}\right) \ldots\left(\forall x_{n}\right)\left(\exists y_{n}\right) \quad \text { player I did not win. }
$$

This, however, is clearly the statement that player II won.

The same proof appears in Jones [Jon1982]. The proof is valid only for games whose number of moves is bounded by a constant, otherwise the negation doesn't necessarily proclaim that player II won. A finite number of moves isn't good enough. Steinhaus proposed to make Theorem * an axiom for the case of infinite play, so there wouldn't be any draws: play would always terminate in a finite, if unbounded, number of moves, with precisely one of the two players winning. Infinite play was also treated by Gale and Stewart [GaS1953] where it was shown that both players may have a winning strategy if the axiom of choice, rather than Steinhaus' axiom, is adopted. Their games are somewhat different; the outcome is determined by the concatenation of the (infinite number of) moves, rather than by reaching a set of terminals. No draws are considered there.

Theorem 1 implies that every position in a game without ties has a unique $P-, N$ - or $D$-label. If the game-graph of $\Gamma$ is finite and acyclic, then Theorem 1 reduces to the classical result that every position has a unique $P$ - or $N$-label. The finiteness and acyclicity requirements are sufficient but not necessary; the dichotomy result holds also for certain families of infinite or cyclic games, e.g., for the game of Example 3: even if there are loops on any subsets of the odd-indexed vertices.

How can we compute the $P$-, $N$-, $D$-labels? Can Lemma 1 help? Lemma 1 is unsatisfactory in at least two respects. 
(a) It does not characterize the $P_{-}, N$ - and $D$-labels. See Fig. 2(a), where labels $P$ and $N$ on the two vertices satisfy the conditions of Lemma 1; but so does the labeling of both of them by $D$, and only the latter is the unique labeling satisfying Theorem 1.

(b) The player moving from an $N$-position may find it difficult to consummate a win. A token on the vertex labeled $N$ in Fig 2(b) can indeed be pushed to the leaf, thus realizing a win. However, there is another follower labeled $P$, and going to it is only a nonlosing move. The digraph may be embedded within a large digraph, or the player may have only local information about the label of a vertex and the labels of its immediate followers. In both of these cases it may be nonobvious which $P$-follower of an $N$-position leads to a win.

These two difficulties are connected, and both can be remedied by a single medicine, namely by introducing an associated counter function $c: V \cap \mathcal{P} \rightarrow \mathbb{Z}^{0}$ as done in the following algorithm for computing the $P, N, D$-labels. (For classical games, which are finite and acyclic, these two difficulties do not exist. This is clear for the second (going to any $P$-follower from an $N$-position leads to a win), and can be proved for the first.) We state the algorithm for the case of normal play, which is all that's needed in the sequel. In a way, the counter function fills the function of the subscripts of $P$ in Definition 1.

Algorithm PND for computing the $P_{-}, N$ - and D-positions of a finite cyclic digraph.

1. (Initialize counter.) Put $m \leftarrow 0$.

2. ( $P$-label and counter.) As long as there is an unlabeled vertex $u$ all of whose followers are labeled $N^{\prime}$, label $u$ by $P^{\prime}$ and put $c(u) \leftarrow m, m \leftarrow m+1$.

3. ( $N$-label.) Label by $N^{\prime}$ every unlabeled vertex which has a follower labeled $P^{\prime}$ and return to 2 .

4. ( $D$-label.) Label all unlabeled vertices by $D^{\prime}$. End.

The complexity of this algorithm, which requires examining each edge once, is $O(|E|)$. We have:

Theorem 2. For every finite cyclic digraph, the labels $P^{\prime}, N^{\prime}$ and $D^{\prime}$ assigned by Algorithm PND are $P_{-}, N$ - and D-labels respectively, and the first property of Lemma 1 can be strengthened to:

$$
\begin{aligned}
& u \in P \text { if and only if: } F(u) \subseteq \mathcal{N}, \text { and for every } v \in F(u) \\
& \text { there is } w \in F(v) \cap \mathcal{P} \text { with } c(w)<c(u) .
\end{aligned}
$$

Proof. Labels $P, N, D$ exist uniquely by Theorem 1 . A vertex $u$ is labeled $P^{\prime}$ in step 2 if and only if $F(u) \subseteq \mathcal{N}^{\prime}$ if and only if each $v \in F(u)$ has a follower which had been labeled $P^{\prime}$ at an earlier stage if and only if $P^{\prime}$ has property (1). Also $u$ is labeled $N^{\prime}$ in step 3 if and only if $F(u) \cap P^{\prime} \neq \emptyset$.

Let $u \in \mathcal{D}^{\prime}$. Then $F(u) \cap \mathcal{P}^{\prime}=\emptyset$, since otherwise $u$ would have been labeled $N^{\prime}$ in step 3. Also $F(u) \nsubseteq \mathcal{N}^{\prime}$, otherwise $u$ would have been labeled $P^{\prime}$ in step 2. Thus there is $v \in F(u) \cap \mathcal{D}^{\prime}$, leading to an infinite sequence of $D^{\prime}$-followers. So $u$ satisfies the condition of a $D$-position by Lemma 1. Therefore $\mathcal{P}^{\prime} \subseteq \mathcal{P}, \mathcal{N}^{\prime} \subseteq \mathcal{N}$ and $\mathcal{D}^{\prime} \subseteq \mathcal{D}$. Since step 4 of the algorithm guarantees that every vertex gets precisely one label, it follows that $V=\mathcal{P}^{\prime} \cup \mathcal{N}^{\prime} \cup \mathcal{D}^{\prime}$; and $V=\mathcal{P} \cup \mathcal{N} \cup \mathcal{D}$ follows from Theorem 1. Hence $\mathcal{P}^{\prime}=\mathcal{P}, \mathcal{N}^{\prime}=\mathcal{N}$ and $\mathcal{D}^{\prime}=\mathcal{D}$.

We now collect together those properties of the $P_{-}, N$ - and $D$-labels whose acquaintance we have already made, and reintroduce infinite digraphs. 
Corollary 2. Let $G=(V, E)$ be a any cyclic game-graph, not necessarily finite, which has a counter function $c: V \cap \mathcal{P} \rightarrow \mathcal{O}$ satisfying (1), without ties. Then there is a unique partition: $V=\mathcal{P} \cup \mathcal{N} \cup \mathcal{D}$ such that:

(i) $u \in \mathcal{P}$ if and only if $F(u) \subseteq \mathcal{N}$,

(ii) $u \in \mathcal{N}$ if and only if $F(u) \cap \mathcal{P} \neq \emptyset$,

(iii) $u \in \mathcal{D}$ if and only if $F(u) \cap \mathcal{P}=\emptyset$ and $F(u) \cap \mathcal{D} \neq \emptyset$.

Proof. A unique partition $V=\mathcal{P} \cup \mathcal{N} \cup \mathcal{D}$ exists by Theorem 1 independently of Algorithm PND, which applies only to finite $G$; property (i) is included in (1), and properties (ii) and (iii) are the last two assertions of Lemma 1.

Infinite digraphs with a counter function $c$ satisfying (1) do exist. Thus in Example 3, $c(2 i)=i$ for all $i \geq 0$ satisfies (1). Also for Example 4 an appropriate counter function satisfying[(1) can be defined.

Corollary 3. Let $G$ be as in Corollary 2. A player moving from an $N$-position can consummate a win by always moving to a $P$-follower of minimum counter function value.

Proof. Follows from property (1): the winner can arrange that any two consecutive $P$-positions $u, w$ will satisfy $c(w)<c(u)$. Since every set of ordinals is well-ordered and so in particular totally-ordered (see e.g., Halmos [Hal1960] $\$ 20$ ), the winner will reach a leaf in a finite number of moves.

We have thus remedied shortcoming (b) mentioned above. For example, in Fig. 2(b), the leaf will get a lower counter function value than the other vertex labeled $P$, if the labeling is done by Algorithm PND. Moreover, also shortcoming (a) has disappeared as will be shown now. Obviously the counter function $c$ is not unique, but properties (i), (ii), (iii) of the $P-, N$ - and $D$-labels of Corollary 2 provide a characterization for these labels. Note that the $P$ - and $N$-labels in Fig. 2(a) are inconsistent with property $(1)$.

Theorem 3. Let $G=(V, E)$ be a cyclic digraph, not necessarily finite, which has a counter function $c: V \cap \mathcal{P} \rightarrow \mathcal{O}$ satisfying (1). Suppose there is a partition $V=\mathcal{P}^{\prime} \cup \mathcal{N}^{\prime} \cup \mathcal{D}^{\prime}$ where $\mathcal{P}^{\prime}$, $\mathcal{N}^{\prime}, \mathcal{D}^{\prime}$ satisfy the three conditions of Corollary 2. Then $\mathcal{P}^{\prime}=\mathcal{P}, \mathcal{N}^{\prime}=\mathcal{N}, \mathcal{D}^{\prime}=\mathcal{D}$.

Proof. By Theorem 1 there is a unique partition $V=\mathcal{P} \cup \mathcal{N} \cup \mathcal{D}$. Let

$$
T=\left\{u \in V: u \in \mathcal{P}^{\prime}, u \notin \mathcal{P}\right\} .
$$

Pick $u \in T$ with $c^{\prime}(u)$ minimum, where $c^{\prime}$ is a counter function: $V \cap \mathcal{P}^{\prime} \rightarrow \mathcal{O}$. By Theorem 1, $u \in \mathcal{N} \cup \mathcal{D}$.

Assume first $u \in \mathcal{N}$. Then property (ii) of Corollary 2 implies that there is $v \in F(u) \cap \mathcal{P}$. By property (i), $v \in \mathcal{N}^{\prime}$, and there is $w \in F(v) \cap \mathcal{P}^{\prime}$ with $c^{\prime}(w)<c^{\prime}(u)$ (see Fig. 4). Furthermore, $w \in N$ by property (i). Thus also $w \in T$, contradicting the minimality of $c^{\prime}(u)$.

Secondly, assume that $u \in \mathcal{D}$. By property (iii), there is $v \in F(u) \cap \mathcal{D}$. By property (i), $v \in \mathcal{N}^{\prime}$, and there is $w \in F(v) \cap \mathcal{P}^{\prime}$ with $c^{\prime}(w)<c^{\prime}(u)$. Since $v \in \mathcal{D}$, property (iii) implies $w \notin \mathcal{P}$. Thus also $w \in T$, which is the same contradiction as in the previous case. Therefore $T=\emptyset$.

If $u \in \mathcal{N} \cap \mathcal{D}^{\prime}$, then $u$ has a $P=P^{\prime}$-follower, contradicting property (iii) for $\mathcal{D}^{\prime}$. Similarly for $u \in \mathcal{N}^{\prime} \cap \mathcal{D}$. Thus also $\mathcal{N}^{\prime}=\mathcal{N}$ and $\mathcal{D}^{\prime}=\mathcal{D}$. 


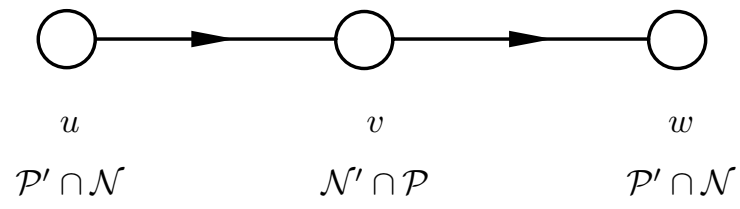

Figure 4. An impossible situation.

Since Lemma 1 is so fundamental for game theory, we exhibit below, very briefly, another approach for establishing it, that of fixpoint logic, also known as $\mu$-Calculus. See e.g., Kozen [Koz1983]. Let $f$ be a logical relation. We are looking for a solution in a predicate or set $S$ of $S=f(S)$. If it exists, it is called a fixed point of $f$. We write $\mu S . f(S)$ for the minimal solution of $S=f(S)$, and $\nu S . f(S)$ for its maximal solution; minimal and maximal in the sense of set inclusion.

Definition 2. Given a game $\Gamma$ without ties which may contain cycles or loops, or may be infinite, with arbitrary termination set $\tau$. Player I begins to play from position $u_{0}$ in $\Gamma$. Then $u_{0}$ is a $P$-position if

$$
\mu P\left(u_{0}\right) . \forall u_{1} \in F\left(u_{0}\right) \exists u_{2} \in F\left(u_{1}\right) P\left(u_{2}\right) .
$$

The position $u_{0}$ is an $N$-position if

$$
\mu N\left(u_{0}\right) . \exists u_{1} \in F\left(u_{0}\right) \forall u_{2} \in F\left(u_{1}\right) N\left(u_{2}\right) .
$$

The position $u_{0}$ is a $D$-position if

$$
\nu D\left(u_{0}\right) . \exists u_{1} \in F\left(u_{0}\right) D\left(u_{1}\right) \wedge \forall u_{1} \in F\left(u_{0}\right)\left(D\left(u_{1}\right) \vee N\left(u_{1}\right)\right) .
$$

The relations (2)-(4) are clearly monotonic, i.e., viewing them, say (2), as a function $f$ of the predicate $P: f(P)=\forall u_{1} \in F\left(u_{0}\right) \exists u_{2} \in F\left(u_{1}\right) P\left(u_{2}\right)$, we have, $\forall P_{1} \forall P_{2} \forall u \in V: P_{1}(u) \rightarrow P_{2}(u) \Rightarrow$ $f\left(P_{1}\right) \rightarrow f\left(P_{2}\right)$. Hence by the Tarski-Knaster fixpoint Theorem [Tar1955], there is a solution to (2)-(4).

Analogously to Lemma 1 we prove,

Lemma 2. Let $G=(V, E)$ be a cyclic, possibly infinite, game-graph without ties. Then for every $u=u_{0} \in V$ we have,

relation (2) holds if and only if

$$
\mu P\left(u_{0}\right) . \forall u_{1} \in F\left(u_{0}\right) N\left(u_{1}\right)
$$

relation (3) holds if and only if

$$
\mu N\left(u_{0}\right) . \exists u_{1} \in F\left(u_{0}\right) P\left(u_{1}\right) .
$$

Proof. Let $u_{0} \in \tau$. Then $u_{0} \in \mathcal{P}$ by (2), which is satisfied vacuously in this case, and by the fact that the first part of (4) doesn't hold. Moreover, (5) is clearly satisfied vacuously for $u_{0}$. Now 
let $u_{0}$ be any predecessor of some $u_{1} \in \tau$. By $(3), u_{0} \in \mathcal{N}$, since $\forall u_{2} \in F\left(u_{1}\right) N\left(u_{2}\right)$ holds vacuously if $u_{1} \in \tau$, and since the second part of (4) doesn't hold. Thus (6) holds for $u_{0}$.

Suppose, inductively, that the lemma holds for all $u \in \mathcal{N} \cup \mathcal{P}$ which lie on any directed path from some $u_{0}=u$.

(i) Let $u_{0} \in \mathcal{P}$. By (2) and the induction hypothesis (5) applied to $P\left(u_{2}\right)$,

$$
\mu P\left(u_{0}\right) . \forall u_{1} \in F\left(u_{0}\right)\left[\exists u_{2} \in F\left(u_{1}\right) \forall u_{3} \in F\left(u_{2}\right) N\left(u_{3}\right)\right] .
$$

The part in brackets is (3) for $u_{1}$, establishing (5).

(ii) Let $u_{0} \in \mathcal{N}$. By (3) and the induction hypothesis (6) applied to $N\left(u_{2}\right)$,

$$
\mu N\left(u_{0}\right) . \exists u_{1} \in F\left(u_{0}\right)\left[\forall u_{2} \in F\left(u_{1}\right) \exists u_{3} \in F\left(u_{2}\right) P\left(u_{3}\right)\right] .
$$

The part in brackets is (2) for $u_{1}$, establishing (6).

Conversely, suppose that (5) and (6) hold. Substituting (6) into (5) gives (2); and (5) into (6) gives (3).

Finally, we show that the $P_{-}, N_{-}, D$-labels of Definitions 1 and 2 are the same. Denote those of Definition 1 by $P^{\prime}, N^{\prime}, D^{\prime}$, and their sets by $\mathcal{P}^{\prime}, \mathcal{N}^{\prime}, \mathcal{D}^{\prime}$.

Theorem 4. Let $G=(V, E)$ be a cyclic digraph, not necessarily finite, which has a counter function $c: V \cap \mathcal{P} \rightarrow \mathcal{O}$ satisfying (1). Then $\mathcal{P}=\mathcal{P}^{\prime}, \mathcal{N}=\mathcal{N}^{\prime}, \mathcal{D}=\mathcal{D}^{\prime}$.

Proof. Using the method of Theorem 3 one can show easily that $\mathcal{P}^{\prime} \subseteq \mathcal{P}$ and $\mathcal{N}^{\prime} \subseteq \mathcal{N}$. If any of these inclusions would be strict, then we would have $\mathcal{D}^{\prime} \supset \mathcal{D}$ strictly, contradicting the maximality of the set $\mathcal{D}$ as expressed in (4).

The theory of (impartial) cyclic games is due to Smith [Smi1966] see also[Con1976] ch. 11, pp. 133-135, [BCG1982] ch. 12. An algorithm similar to Algorithm PND appears in the proof of Theorem 2 in [Cnd1992], but no counter function is mentioned there. Algorithm PND is a specialization of Algorithm G in [FrY1986] p. 168, for computing the generalized Sprague-Grundy function $\gamma$ of a digraph, which has complexity $O(|V||E|)$. The higher complexity is due to the fact that in the latter algorithm we seek out and label grandparents of previously labeled grandchildren, rather than parents of previously labeled children, as in the former algorithm. In fact, $\gamma$ is a generalization of the $P-, N$-, $D$-labeling. One can trade a counter function, which appears in Definitions 1 and 2 there, by the requirement of the maximality of the set of infinities of $\gamma$ in Definition 3 there. This is analogous to the present case where a counter function $c$, as used in Algorithm PND, can be traded for a maximality operator $\nu$ in Definition 2, though $\nu$ seems to cure only shortcoming (a) above, not (b) For related treatments of games using fixpoint logic see e.g., Banaschewski and Pultr [BaP1991] and Stirling $[\mathbf{S t i} \geq \mathbf{1 9 9 6}]$. No draw positions were considered by them.

\section{The Structure of Digraph Kernels}

We are now ready to state our results on digraphs precisely, and to prove them by using the game-theoretic results obtained in the previous section.

Theorem 5. Let $G=(V, E)$ be any cyclic digraph, not necessarily finite, which has a counter function $c: V \cap \mathcal{P} \rightarrow \mathcal{O}$ satisfying property (1). Then $\mathcal{P}$ is a subset of every kernel and $\mathcal{N}$ is a subset 
of the complement of every kernel; the kernels (and their complements) are possibly nonunique only on $\mathcal{D}$.

Proof. By Theorem 1, the vertex set $V$ of $G$ can be partitioned uniquely into $\mathcal{P}, \mathcal{N}$ and $\mathcal{D}$. Denote by $\mathcal{K}$ the set of all kernels of $G$. Then the first part of the claim is equivalent to: $\mathcal{P} \subseteq$ $K \forall K \in \mathcal{K}$ and $\mathcal{N} \subseteq V-K \forall K \in \mathcal{K}$. We see that this is satisfied vacuously if $G$ has no kernel $(\mathcal{K}=\emptyset)$. If $\mathcal{P}=\emptyset$, then $\mathcal{N}=\emptyset$, and then the first part holds trivially. The last part of the claim clearly holds for these two special cases. If the claim is false, we may thus suppose that there exists $u \in \mathcal{P}, u$ not in some kernel $K$. Let $T=\{u \in \mathcal{P}: u \notin K\}$. If $T$ is nonempty, there exists $u \in T$ with $c(u)$ minimum; it exists by the well-ordering principle. Since $u \notin K$, there exists $v \in F(u) \cap K$. By Lemma 1. $v \in \mathcal{N}$ and there exists $w \in F(v) \cap \mathcal{P}$ with $c(w)<c(u)$. We have $w \notin K$, and so $w \in T$, contradicting the minimality of $c$. Thus $\mathcal{P}$ is in every kernel. It follows that $\mathcal{N}$ is in the complement of every kernel. Hence the only subset on which the kernel is possibly nonunique, is $\mathcal{D}$.

Note the simplicity of the proof, once we set up the appropriate tools. Theorem 5 says that $\mathcal{P}$ is a set of fixed points for all the kernels and $\mathcal{N}$ is a set of fixed points for the complement of every kernel. But it doesn't yet tell the full story. We now show that every kernel of $G$ can be written as a union of 2 kernels, one of them fixed.

Theorem 6. (Decomposition Theorem.) Let $G=(V, E)$ be any cyclic digraph, not necessarily finite, which has a counter function $c: V \cap \mathcal{P} \rightarrow \mathcal{O}$ satisfying (1). Let $G_{1}=\left(V_{1}, E_{1}\right)$ be the subgraph induced by the vertex subset $\mathcal{P} \cup \mathcal{N}$, and $G_{2}=\left(V_{2}, E_{2}\right)=G-G_{1}$ the subgraph induced by the vertex subset $\mathcal{D}$. Then $G_{1}$ has a unique kernel $K\left(G_{1}\right)=V_{1} \cap \mathcal{P}$; it is the empty set if and only if $\mathcal{P}=\emptyset$. Let $\mathcal{K}, \mathcal{K}_{2}$ denote the set of kernels of $G, G_{2}$ respectively. Then $\mathcal{K}=K\left(G_{1}\right) \cup \mathcal{K}_{2}=\left\{K\left(G_{1}\right) \cup K\left(G_{2}\right): K\left(G_{2}\right) \in \mathcal{K}_{2}\right\}$. In particular, $\mathcal{K}=\emptyset$ if and only if $\mathcal{K}_{2}=\emptyset$.

Proof. The partition $V=\mathcal{P} \cup \mathcal{N} \cup \mathcal{D}$ exists and is unique by Theorem 1. The set $S:=V_{1} \cap \mathcal{P}$ is clearly a kernel of $G_{1}$. Thus by Theorem 5, $S$ is in every kernel of $G_{1}$. Since all the remaining vertices of $G_{1}$ are in in $V_{1} \cap \mathcal{N}$ and $S$ is dominating, $S$ is the unique kernel of $G_{1}$. The digraph $G_{1}$ and its kernel $K\left(G_{1}\right)$ are empty if and only if $\mathcal{P}=\emptyset$.

Let $u$ be any vertex of $V_{2}$. If it has a follower $v \in V_{1}$, then $v \in \mathcal{N}$ by Lemma 1 . If $u \in V_{2}$ has a predecessor $w \in V_{1}$, then again $w \in \mathcal{N}$, since $w \in \mathcal{P}$ implies $F(w) \subseteq \mathcal{N}$ by Lemma 1 , but $u \in F(w) \cap \mathcal{D}$. Thus the "boundary" of $G_{1}$ has no influence on the structure of any kernel of $G_{2}$. Conversely, if $(u, v) \in E, u \in V_{1}, v \in V_{2}$ and $v$ is in a kernel of $G_{2}$, the edge $(u, v)$ cannot affect the kernel $K\left(G_{1}\right)$, since it is unique. We conclude that the kernel $K\left(G_{1}\right)$ is isolated from any kernel $K\left(G_{2}\right)$ : they cannot influence each other adversely when embedded together in $G$, as would be the case in the presence of an edge of $G$ joining a vertex of $G_{2}$ and a vertex of $G_{1}$ labeled $P$. Therefore any kernel $K\left(G_{2}\right) \in \mathcal{K}_{2}$ can be adjoined to $K\left(G_{1}\right)$ to form a kernel $K=K\left(G_{1}\right) \cup K\left(G_{2}\right)$ of $G$; and conversely, every kernel $K$ of $G$ can be decomposed into the kernel $K\left(G_{1}\right)$ and a kernel $K\left(G_{2}\right) \in \mathcal{K}_{2}$.

Note that normally a union of 2 kernels of 2 induced subgraphs of a connected digraph is not a kernel of the entire digraph, because edges connecting the 2 induced subgraphs influence these kernels adversely. Informally, if we consider a digraph with a multiplicity of kernels to be "malignant", then Theorem 6 indicates a healing process: the cancer is completely contained in $\mathcal{D}$; removing it preserves 
the healthy part $\mathcal{P} \cup \mathcal{N}$, though the healthy part may be empty, as is the case for the digraph of Fig. 1. But for other digraphs, the malignant part may be empty! On the other hand, if we do have to compute or estimate the number of kernels, then Theorem 6 hows that it suffices to count or estimate this number on the subgraph $G_{2}$, which is computationally more efficient, in general, than doing it on $G$ itself.

If $G$ is finite, then $\mathcal{P}, \mathcal{N}, \mathcal{D}$ can be computed in polynomial time by Algorithm PND. We combine Theorems 5 and 6 for this case.

Corollary 4. Let $G=(V, E)$ be a connected finite cyclic digraph. Let $G_{1}=\left(V_{1}, E_{1}\right)$ be the subgraph induced by the vertex subset $\mathcal{P} \cup \mathcal{N}$, and $G_{2}=\left(V_{2}, E_{2}\right)=G-G_{1}$ the subgraph induced by the vertex subset $\mathcal{D}$. Then $G_{1}$ has a unique kernel $K\left(G_{1}\right)=V_{1} \cap \mathcal{P}$; it is the empty set if and only if $\mathcal{P}=\emptyset$. Let $\mathcal{K}, \mathcal{K}_{2}$ denote the set of kernels of $G, G_{2}$ respectively. Then $\mathcal{K}=K\left(G_{1}\right) \cup \mathcal{K}_{2}=$ $\left\{K\left(G_{1}\right) \cup K\left(G_{2}\right): K\left(G_{2}\right) \in \mathcal{K}_{2}\right\}$. In particular, $\mathcal{K}=\emptyset$ if and only if $\mathcal{K}_{2}=\emptyset$. Moreover, the decomposition of $G$ into $G_{1}$ and $G_{2}$ and the kernel $K\left(G_{1}\right)$ can be computed in $O(|E|)$ steps.

Proof. Since for a connected digraph, $\mathcal{P}, \mathcal{N}, \mathcal{D}$ can be computed in $O(|E|)$ steps, the result follows from Theorem 2 .

Corollary 4 implies, informally, that the source of the NP-completeness of the existence of a digraph-kernel and the \#P-completeness result of their number, are both contained in $\mathcal{D}$.

There may be a unique kernel even when there are $D$-positions. A simple example is depicted in Fig. 5 ; the 2 vertices of degree 2 constitute a unique kernel.

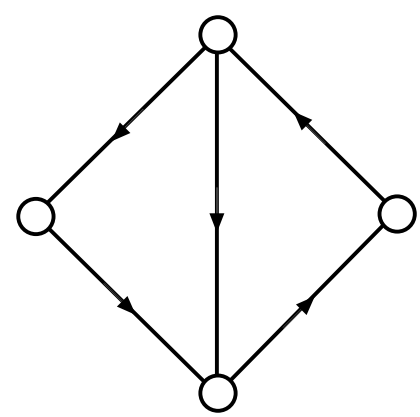

FiguRE 5 . Unique kernel for the case $\mathcal{D}=V$.

As an example of Corollary 4, we may ask about the existence of kernels in the digraph depicted in Fig. 6. To answer that, we first apply Algorithm PND, which then enables us to decompose $G$ into the induced digraphs $G_{1}, G_{2}$ (Fig. 7). It is easily seen that $G_{2}$ has no kernel, so $G$ has none. (It suffices to reverse the direction of a single edge of $G_{2}$ (which?), and correspondingly of $G$, to get a unique kernel.)

We don't know a priori whether $K$ is unique on $D$ or not, and we can't find out for a general digraph in polynomial time, unless $N P=P$. But we can give a game-theoretic interpretation to digraphs whose kernels intersect the "uncertainty region" $\mathcal{D}$ : a token-pushing game on them, in 


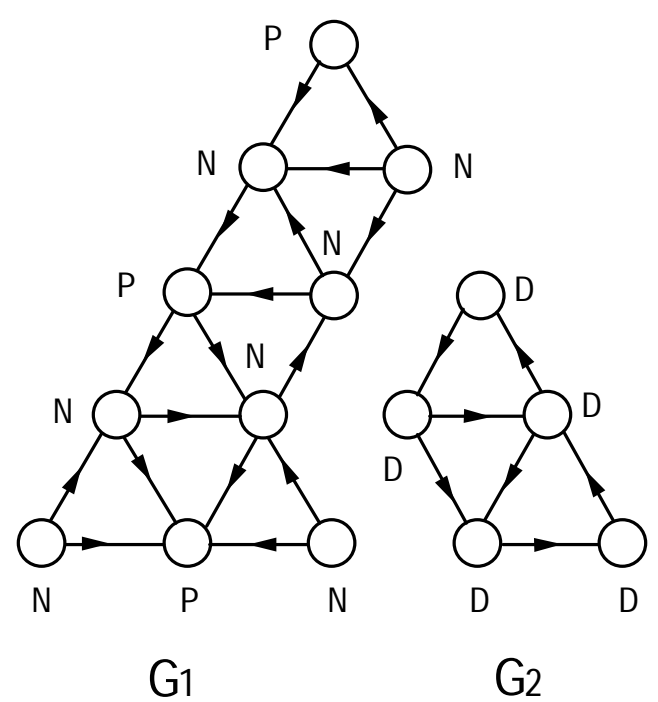

Figure 6. How many kernels does this digraph have?

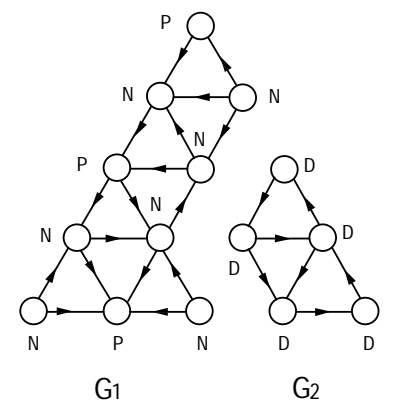

FiguRe 7. Answer: it suffices to analyse $G_{2}$.

normal play, has $D$-positions, all of them lying in $\mathcal{D}$. In fact, in the example of Fig. 5 , the kernel is not a set of $P$-positions. Define a strategy kernel to be a kernel in a digraph $G$ all of whose elements are $P$-positions in normal play of a game with game-graph $G$.

Theorem 7. Let $G=(V, E)$ be a finite cyclic digraph. Then $G$ has a strategy kernel $K=\{u \in$ $V: u \in \mathcal{P}\}$ if and only if $\mathcal{D}=\emptyset$.

Proof. If $G$ has a strategy kernel $K$, then $\mathcal{D}=\emptyset$, since any element of $\mathcal{D}$ is neither in $K$ nor in its complement. If $\mathcal{D}=\emptyset$, then $V$ is partitioned uniquely into $\mathcal{P}$ and $\mathcal{N}$, so the result follows from Theorem 5 . 
Corollary 5. If a finite cyclic digraph $G$ has a strategy kernel, it is a unique kernel of $G$.

Proof. A digraph cannot have 2 distinct strategy kernels by Theorem 1.

Can one produce a decomposition similar to that of Theorem 6 also when $\mathcal{P}=\emptyset$, i.e., all vetices are in $\mathcal{D}$ ? We mention briefly that for a special family of digraphs the answer is positive. A slight generalization of the problem of stable marriages, see Gale and Shapley [GaS1962], can be cast as a digraph $G$ on a grid with horizontal and vertical edges only, and every row and every column is acyclic. This generalization is used e.g., in Galvin's proof [Gal1995] of the Dinitz conjecture, mentioned in a paper by Erdös, Rubin and Taylor [ERT1980]. Stable marriages correspond to kernels in $G$, which has no leaf unless there is a pair $(m, w)$ such that the woman $w$ is the first choice of the man $m$ and $m$ is the first choice of $w$. The algorithm in which the men propose first results in marriages in which the men get their best choices, and the women their worst. Conversely if the women propose first. Knowing these two extreme cases, one can partition the vertex set into the subsets belonging to no kernel, every kernel, and the rest.

Conclusion. We have constructed game-theoretic tools, of independent interest, and applied them to study the structure of digraph kernels. There exist more sophisticated tools, to provide polynomial strategies for the more complicated class of annihilation games: a finite number of tokens is placed initially on a subset of the vertices, at most 1 token per vertex. A move consists of selecting a token and sliding it to a follower. If that follower was occupied, then both tokens are phased out of the game (annihilation). These tools enable to shed more light on digraph kernels and their relationship to certain homomorphism kernels. This in turn can be applied to obtain further results in the theory of linear error-correcting codes. Some of this is planned to appear in [Fra $\geq \mathbf{1 9 9 7}]$

Acknowledgment. Herb Wilf pointed out the desirability of a formal definition of the $\mathrm{P}_{-}, \mathrm{N}-$ and $D$-positions. Ron Holzman and Donald A. Martin made useful comments on a previous version of Definition 1 The present Definition 1 is a slight variation of a suggestion made to me by Azriel Levy. Amir Pnueli introduced me to fixpoint logic, which led to Definition 2 and Lemma 2. Gil Kalai told me about partitioning the stable marriage digraph into subsets of kernels and Uri Zwick pointed out the relationship with reference [Cnd1992]. Many thanks to all of them!

\section{References}

1. [BaP1991] B. Banaschewski and A. Pultr [1991], Tarski's fixpoint lemma and combinatorial games, Order 7, 375-386.

2. [Ber1992] C. Berge [1992], Graphs (Chapter 4), Second Edition, Elsevier (French: Gauthier Villars 1988).

3. [BCG1982] E. R. Berlekamp, J. H. Conway and R. K. Guy [1982], Winning Ways (two volumes), Academic Press, London.

4. [BrP1993] R. A. Brualdi and V. S. Pless [1993], Greedy codes, J. Combin. Theory (Ser. A) 64, 10-30.

5. [Chv1973] V. Chvátal [1973], On the computational complexity of finding a kernel, Report No. CRM-300, Centre de Recherches Mathématiques, Université de Montréal.

6. [Cnd1992] A. Condon [1992], The complexity of Stochastic games, Information and Computation 96, 203-224. 
7. [Con1976] J. H. Conway [1976], On Numbers and Games, Academic Press, London.

8. [Con1990] J. H. Conway [1990], Integral lexicographic codes, Discrete Math., 83, 219-235.

9. [CoS1986] J. H. Conway and N. J. A. Sloane [1986], Lexicographic codes: error-correcting codes from game theory, IEEE Trans. Inform. Theory IT-32, 337-348.

10. [ERT1980] P. Erdös, A. L. Rubin and H. Taylor [1980], Choosability in graphs, Congr. Numer. 26, 122-157.

11. [Fra1981] A. S. Fraenkel [1981], Planar kernel and Grundy with $d \leq 3, d_{\text {out }} \leq 2, d_{\text {in }} \leq 2$ are NP-complete, Discrete Appl. Math. 3, 257-262.

12. [Fra1996] A. S. Fraenkel [1996], Error-correcting codes derived from combinatorial games, in: Games of No Chance, Proc. MSRI Workshop on Combinatorial Games, July, 1994, Berkeley, CA (R. J. Nowakowski, ed.), MSRI Publ. Vol. 29, Cambridge University Press, Cambridge, pp. 417-431.

13. [Fra $\geq \mathbf{1 9 9 7}]$ A. S. Fraenkel $[\geq 1997]$ Adventures in Games and Computational Complexity, Graduate Studies in Math., Amer. Math. Soc., to appear.

14. [FrY1986] A. S. Fraenkel and Y. Yesha [1986], The generalized Sprague-Grundy function and its invariance under certain mappings, J. Combin. Theory (Ser. A) 43, 165-177.

15. [GaS1962] D. Gale and L. S. Shapley [1962], College admissions and the stability of marriages, Amer. Math. Monthly 69, 9-15.

16. [GaS1953] D. Gale and F. M. Stewart [1953], Infinite games with perfect information, Contributions to the Theory of Games, Ann. of Math. Stud. 2(28), 245-266, Princeton.

17. [Gal1995] F. Galvin [1995], The list chromatic index of a bipartite multigraph, J. Combin. Theory (Ser. B) 63, 153-158.

18. [Hal1960] P. R. Halmos [1960], Naive Set Theory, Van Nostrand, Princeton, NJ.

19. [Jon1982] J. P. Jones [1982], Some undecidable determined games, Internat. J. Game Theory 11, 63-70.

20. [JFr1995] J. P. Jones and A. S. Fraenkel [1995], Complexities of winning strategies in diophantine games, J. Complexity 11, 435-455.

21. [Kac1974] M. Kac [1974], Hugo Steinhaus, a reminiscence and a tribute, Amer. Math. Monthly 81, 572-581 (p. 577).

22. [Koz1983] D. Kozen [1983], Results on the propositional $\mu$-calculus, Theoret. Comput. Sci. 27, 333-354.

23. [Rab1957] M. O. Rabin [1957], Effective computability of winning strategies, Contributions to the Theory of Games vol. 3, Ann. of Math. Stud. 39, 147-157, Princeton.

24. [Ric1953] M. Richardson [1953], Solutions of irreflexive relations, Ann. of Math. 58, 573590.

25. [Smi1966] C. A. B. Smith [1966], Graphs and composite games, J. Combin. Theory 1, 51-81. Reprinted in slightly modified form in: A Seminar on Graph Theory (F. Harary, ed.), Holt, Rinehart and Winston, New York, NY, 1967.

26. [Sti $\geq \mathbf{1 9 9 6}]$ C. Stirling [1996], Model checking and other games, presented at Computer Science Logic, September, 1996.

27. [SzC1994] J. L. Szwarcfiter and G. Chaty [1994], Enumerating the kernels of a directed graph with no odd circuits, Inform. Process. Lett. 51, 149-153.

28. [Tar1955] A. Tarski [1955], A lattice theoretical fixpoint theorem and its applications, Pacific J. Math. 5, 285-309. 
29. [VLe1976] J. van Leeuwen [1976], Having a Grundy-numbering is NP-complete, Report No. 207, Computer Science Dept., Pennsylvania State University, University Park, PA.

30. [VNe1928] J. von Neumann [1928], Zur Theorie der Gesellschaftsspiele, Math. Ann. 100, $295-320$.

31. [VNM1953] J. von Neumann and O. Morgenstern [1953], Theory of Games and Economic Behaviour, 3rd ed., Princeton University Press, Princeton, NJ.

32. [Zer1912] E. Zermelo [1912], Über eine Anwendung der Mengenlehre auf die Theorie des Schachspiels, Proc. 5th Int. Cong. Math., Cambridge, Cambridge University Press, 1913, Vol. II, pp. 501-504. 